Classification

Physics Abstracts

$41.10 \mathrm{~F}-89.20-02.70$

\title{
Modélisation de systèmes électrotechniques par couplage des équations électriques et magnétiques
}

\author{
F. Hecht $\left({ }^{1}\right)$, A. Marrocco $\left({ }^{1}\right)$, F. Piriou $\left({ }^{2}\right)$ et A. Razek $\left({ }^{2}\right)$ \\ (1) INRIA Domaine de Voluceau, Rocquencourt BP 105, 78153 Le Chesnay, France \\ (2) LGEP, Plateau du Moulon, 91190 Gif sur Yvette, France
}

(Reçu le 10 novembre 1989, accepté le 12 mars 1990)

\begin{abstract}
Résumé. - Pour modéliser des systèmes électromagnétiques en tenant compte du couplage entre les circuits magnétiques et électriques, on est amené à résoudre un système d'équations différentielles spatio-temporelles. En ayant recours à une discrétisation spatiale de type éléments finis et une discrétisation temporelle de type pas-à-pas, on aboutit à un système d'équations algébriques, dont les inconnues sont les valeurs nodales du potentiel vecteur et les courants. A ce niveau, deux approches peuvent être envisagées; dans la première on conserve comme inconnues le potentiel vecteur et les courants, dans la seconde on se ramène à un système d'équations où les inconnues sont uniquement les valeurs nodales du potentiel vecteur. Dans cet article les deux stratégies sont développées ainsi que leurs influences sur le système matriciel à résoudre. Les résultats obtenus pour diverses applications sont donnés avec des comparaisons entre le calcul et l'expérience.
\end{abstract}

\begin{abstract}
For the numerical simulation of electromagnetic systems taking into account coupling of magnetic and electric equations, we have to solve a spatio-temporal differential equation system. Using a spatial discretization by finite element and step-by-step temporal discretization, we obtain an algebric equation system where the unknowns are the vector potential and the currents. At this stage, two approaches can be used. In the first approach, the vector potential and the currents are kept as unknowns. In the second one, the equation system is transformed in such a way that the unknown to be considered is the vector potential only. In this paper the two approaches are considered as well as the influence on the matrix system to solve. Different applications are done and comparisons between numerical results and experimental measurements are given.
\end{abstract}

\section{Introduction.}

Pour la modélisation numérique d'un ensemble comprenant un circuit électrique et un circuit magnétique, deux stratégies peuvent être utilisées.

La première consiste à représenter la partie magnétique par un schéma équivalent dont les éléments sont obtenus en résolvant les équations magnétiques linéarisées autour d'un point de fonctionnement [1]. Un programme de simulation des circuits permet alors de modéliser l'ensemble. Une telle méthode simplifie les problèmes mais son domaine d'applications reste limité. En effet lorsque l'interaction entre les circuits magnétique et électrique devient importante, cette technique perd de sa fiabilité car les équations magnétiques sont découplées des équations électriques.

La seconde stratégie, consiste à simuler le système électromagnétique par un modèle où les équations magnétiques et électriques sont considérées simulta- nément $[3,9,13,14]$. Pour effectuer une telle modélisation, on est amené à résoudre un système d'équations aux dérivées partielles non linéaire couplé à un système d'équations qui peut être également non linéaire. En ayant recours à une discrétisation spatiale de type éléments finis et une discrétisation temporelle, on aboutit à un système d'équations où

électrique et les valeurs nodales du potentiel vecteur aux nœuds du maillage. A ce stade de la modélisation, deux approches peuvent être développées. Dans la première on conserve l'ensemble des inconnues (courants et potentiel vecteur). Dans la seconde on se ramène à un système d'équations où les inconnues sont uniquement les valeurs nodales du potentiel vecteur.

Dans cet article, nous allons traiter le cas de la résolution simultanée et développer les différents calculs qui conduisent à un système d'équations 
algébriques non linéaires. Ensuite nous étudierons les deux approches proposées et leur influence sur le système matriciel à résoudre.

Comme exemple d'application, nous allons présenter les résultats obtenus pour un dispositif électromagnétique fixe (bobine à noyau ferromagnétique) et mobile (machines électriques). Dans ces exemples les résultats obtenus à partir de la simulation seront comparés aux résultats expérimentaux.

\section{Formulation du problème et résolution.}

La modélisation d'un système électromagnétique (voir par exemple [2, 6]) dans le cas de l'approximation bidimensionnelle conduit classiquement à la résolution de l'équation :

$$
\sigma \frac{\partial A}{\partial t}+\operatorname{rot}(\nu \operatorname{rot} A)-J=0
$$

sur le domaine $\Omega \subset \mathbb{R}^{2}$, en tenant compte des conditions aux limites. Dans cette expression $\sigma$ représente la conductivité, $A \equiv(0,0, A)$ le potentiel vecteur, $\nu$ la réluctivité, et $J \equiv(0,0, J)$ la densité de courant.

Dans le cas de systèmes en mouvement, comme les machines tournantes, par exemple, la prise en compte de ce mouvement peut être faite en résolvant l'équation (1) dans deux repères différents. Dans ces conditions le terme $\sigma(v \wedge \operatorname{rot} A)$ n'apparaît pas explicitement $[7,8]$. Pour modéliser l'interface entre ces deux repères, diverses techniques peuvent être utilisées, bande de mouvement [9], macro-élément $[10,11]$, ligne de glissement $[6,12]$.

La densité de courant dans l'équation (1) peut s'exprimer sous la forme :

$$
J=J_{0}+\sum_{k=1}^{n_{\mathrm{p}}} i_{k} \psi_{k}
$$

où $J_{0}$ représente une densité de courant imposée (source de courant donnée ou densité de courant équivalente dans le cas d'aimants permanents), $i_{k}$ le courant dans la phase (ou branche) $k$, $n_{\mathrm{p}}$ le nombre de phases incluses dans le domaine $\Omega$ et $\psi_{k}$ une fonction de $L^{2}(\Omega)$ définie comme étant la densité de courant dans l'espace, correspondant à un courant de $1 \mathrm{~A}$ dans la phase $k$ (on a évidemment $\psi_{k} \equiv 0$ en dehors du support de la phase $k$ ).

Les équations du circuit électrique peuvent s'écrire pour les branches $k, 1 \leqslant k \leqslant n_{\mathrm{p}}$, d'extrémités $k_{1}$ et $k_{2}$ sous la forme :

$$
\left(V_{k_{1}}-V_{k_{2}}\right)=R_{k} i_{k}+\frac{\mathrm{d} \Phi_{k}}{\mathrm{~d} t}+\ell_{k} \frac{\mathrm{d} i_{k}}{\mathrm{~d} t}
$$

et pour les autres branches $q, n_{\mathrm{p}}<q \leqslant n_{\mathrm{b}}$, du circuit électrique, pouvant contenir des résistances, des inductances, des générateurs de tension et des composants non linéaires comme les diodes :

$$
\left(V_{q_{1}}-V_{q_{2}}\right)=e_{q}+R_{q} i_{q}+\ell_{q} \frac{\mathrm{d} i_{q}}{\mathrm{~d} t}+\gamma\left(i_{q}\right)
$$

où $n_{\mathrm{b}}$ représente le nombre total de branches du circuit électrique, $\left(V_{k_{1}}-V_{k_{2}}\right)$ ou $\left(V_{q_{1}}-V_{q_{2}}\right)$ la différence de potentiel aux bornes de la branche $k$ ou $q, R_{k}$ ou $R_{q}$ la résistance, $\ell_{k}$ ou $\ell_{q}$ les inductances non prises en compte dans les équations magnétiques, $\Phi_{k}$ le flux à travers un enroulement et $\gamma\left(i_{q}\right)$ la différence de potentiel aux bornes d'une diode. Bien souvent dans les systèmes électrotechniques, le temps d'établissement du courant dans une diode par rapport aux constantes de temps du système étudié est négligeable. Dans ces conditions, la diode peut être modélisée par un circuit équivalent constitué d'une résistance $R_{\mathrm{d}}$ et d'une tension de seuil $E_{\mathrm{s}}$, plus précisément, nous écrivons

$$
\gamma\left(i_{q}\right)=V_{\mathrm{a}}-V_{\mathrm{b}}=R_{\mathrm{d}}\left(i_{q}\right) i_{q}+E_{\mathrm{s}}
$$

avec $R_{\mathrm{d}}\left(i_{q}\right)= \begin{cases}R & \text { si } i_{q}<0 \\ r & \text { sinon }\end{cases}$

avec $R$ forte valeur de résistance et $r$ faible valeur de résistance. Les relations (1)-(3) montrent comment les équations magnétiques et électriques sont couplées (le flux $\Phi_{k}$ dans (3a) pouvant s'exprimer en fonction du potentiel vecteur $A$ ).

2.1 DisCRÉTISATION. - Pour résoudre numériquement le problème, on peut utiliser une discrétisation spatiale par la méthode des éléments finis $\left(P_{1}\right)$ et une discrétisation temporelle avec un schéma de type implicite (Crank-Nicholson, Euler rétrograde, Gear, ...). L'équation (1) dans le cas du schéma d'Euler rétrograde, exprimée sous forme faible sera du type :

solution de

$$
A_{t+\Delta t} \in \mathcal{V}
$$

$$
\begin{aligned}
\frac{1}{\Delta t} \int_{\Omega} \sigma A_{t+\Delta t} \cdot \omega \mathrm{d} x+\int_{\Omega} \nu\left(x,\left|\operatorname{rot} A_{t+\Delta t}\right|^{2}\right) \operatorname{rot} A_{t+\Delta t} \cdot \operatorname{rot} \omega \mathrm{d} x= \\
\quad=\int_{\Omega}\left[J_{0}+\left(\sum_{k=1}^{n_{\mathrm{p}}} i_{k} \psi_{k}\right)\right] \cdot \omega \mathrm{d} x+\frac{1}{\Delta t} \int_{\Omega} \sigma A_{t} \cdot \omega \mathrm{d} x \quad \forall \omega \in V
\end{aligned}
$$


avec $\vartheta$ espace fonctionnel adéquat $\left(i_{k}\right.$ étant considéré à l'instant $t+\Delta t-$ voir ci-après la discrétisation des équations électriques).

L'équation (4) peut être également exprimée sous forme matricielle (voir $[7,8]$ :

$$
\left(\frac{\mathbf{T}}{\Delta t}+\mathbf{S}\right) A_{t+\Delta t}=F_{t+\Delta t}+\frac{\mathbf{T}}{\Delta t} A_{t}
$$

cette équation matricielle est bien entendu non linéaire car les termes de la matrice $\mathbf{S}$ dépendent de $A_{t+\Delta t}$. La correspondance entre les termes intégraux dans (4) et les termes matriciels dans (4bis) est immédiate. Le vecteur $F$ peut s'écrire :

$$
F=F_{0}+\sum_{k=1}^{n_{\mathrm{p}}} F_{k} i_{k}=F_{0}+\mathbf{F J}_{\left(n_{\mathrm{p}}\right)}
$$

$F_{0}$ étant relatif au terme source $J_{0}$ et avec

$$
F_{k}=\left(\int_{\Omega} \psi_{k} \cdot \omega_{\ell} \mathrm{d} x\right)_{\ell=1, n_{\mathrm{s}}}
$$

dans cette dernière expression $n_{\mathrm{s}}$ représente le nombre de degrés de liberté du potentiel vecteur sur le domaine d'étude $\Omega, \omega_{\ell}$ les fonctions de base usuelles des éléments finis $\left(\mathrm{P}_{1}\right)$ et $\psi_{k}$ est approché par :

$$
\begin{array}{ll}
\psi_{k}(x)=0 & \text { si } x \notin \text { phase } k \\
\psi_{k}(x)=\frac{\varepsilon_{n} N_{n}}{S_{n}} & \text { si } x \in \text { phase } k
\end{array}
$$

pour l'encoche $n$ appartenant à la phase $k, \varepsilon_{n}= \pm 1$ suivant le sens du bobinage, $N_{n}$ représente le nombre de spires et $S_{n}$ la section de l'encoche.

En ce qui concerne les équations du circuit électrique, l'application du schéma d'Euler implicite permet d'écrire les équations sous la forme :

$$
\begin{aligned}
\left.\left(V_{k_{1}}-V_{k_{2}}\right)\right|_{t+\Delta t} & =\left.\left(R_{k}+\frac{\ell_{k}}{\Delta t}\right) i_{k}\right|_{t+\Delta t}+ \\
& +\left.\frac{\Phi_{k}}{\Delta t}\right|_{t+\Delta t}-\left.\frac{\ell_{k}}{\Delta t} i_{k}\right|_{t}-\left.\frac{\Phi_{t}}{\Delta t}\right|_{t}
\end{aligned}
$$

ou bien

$$
\begin{aligned}
& \left.\left(V_{q_{1}}-V_{q_{2}}\right)\right|_{t+\Delta t}=\left.e_{q}\right|_{t+\Delta t}+ \\
& +\quad R_{q}+\frac{q}{\Delta t} i_{q}+\left.\gamma\left(i_{q}\right)\right|_{t+\Delta t}-\frac{q}{\Delta t} i_{q} .
\end{aligned}
$$

Dans l'équation (6a), le flux $\Phi_{k}$ engendré dans les enroulements de la phase $k$, peut s'exprimer en fonction du potentiel vecteur par ( $\psi_{k}$ étant défini en $(5 c, 5 d))$ :

$$
\Phi_{k}=h \int_{\Omega} A \cdot \psi_{k} \mathrm{~d} x
$$

$h$ représentant la longueur active des conducteurs suivant la troisième dimension. En remplaçant dans (7) le potentiel vecteur par son expression discrétisée et en tenant compte de (5b) on peut écrire :

$$
\Phi_{k}=h F_{k}^{T} \cdot A
$$

ou bien sous forme matricielle

$$
\Phi_{\left(n_{\mathrm{p}}\right)}=h \mathbf{F}^{T} A
$$

Comme nous l'avons dit précédemment deux approches peuvent être envisagées pour traiter le problème. La première consiste à utiliser la méthode des mailles pour éliminer les différences de potentiel dans les équations (6) et à regrouper les équations (4)-(8). Dans ce cas les inconnues sont les valeurs nodales du potentiel vecteur $A$ et les courants de mailles $i_{\mathrm{m}}$. La deuxième consiste à exprimer les courants $i_{k}$ dans (2) en fonction des $\Phi_{k}$ donc du potentiel vecteur $A$, et à reporter cette expression dans (1). L'équation (1) devient alors intégro-différentielle pour le potentiel vecteur.

2.2 Première APPROCHE. - En utilisant la loi des mailles et en remplaçant $\gamma(i)$ par $R_{\mathrm{d}} i+E_{\mathrm{s}}$, les équations discrétisées (6), peuvent se mettre sous la forme matricielle :

$$
\begin{aligned}
\left.\frac{\Phi_{(\mathrm{m})}}{\Delta t}\right|_{t+\Delta t}+(\mathbf{R} & \left.+\frac{\mathbf{L}}{\Delta t}\right)\left.\mathfrak{J}_{(\mathrm{m})}\right|_{t+\Delta t}= \\
& =E_{(\mathrm{m})}+\left.\frac{\Phi_{(\mathrm{m})}}{\Delta t}\right|_{t}+\left.\frac{\mathbf{L}}{\Delta t} \mathfrak{J}_{(\mathrm{m})}\right|_{t}
\end{aligned}
$$

on notera que la matrice de correspondance CNT, permet d'exprimer les courants de branches en fonction des courants de mailles. Dans ces conditions l'équation (5a) s'écrit :

$$
F=F_{0}+\mathbf{D} J_{(\mathrm{m})}
$$

avec

$$
\mathbf{D}=\sum_{k=1}^{n_{\mathrm{p}}} F_{k}(C N T)_{k}=\mathbf{F} \overline{\mathbf{C N T}}
$$

$(C N T)_{k}$ représentant la k-ième ligne de la matrice CNT et CNT étant la matrice réduite aux $n_{\mathrm{p}}$ premières lignes de CNT.

e a meme açon, e ux (m) ans equa io peut s'écrire en fonction des valeurs nodales du potentiel vecteur sous la forme :

$$
\Phi_{(\mathrm{m})}=\mathbf{G} A
$$

avec

$$
\mathbf{G}=h \sum_{k=1}^{n_{\mathrm{p}}}(C N T)_{k}^{\mathbf{T}} F_{k}^{\mathrm{T}}=h \overline{\mathbf{C N T}}^{\mathrm{T}} \mathbf{F}^{\mathrm{T}}
$$

En regroupant les équations (4), (9), (10), (11), on obtient le système suivant : 


$$
\begin{aligned}
\left(\begin{array}{cc}
\mathbf{S}+\frac{\mathbf{T}}{\Delta t} & -\mathbf{D} \\
\frac{\mathbf{G}}{\Delta t} & \mathbf{R}+\frac{\mathbf{L}}{\Delta t}
\end{array}\right)\left(\begin{array}{c}
\mathbf{A} \\
\mathfrak{J}_{(\mathrm{m})}
\end{array}\right)_{t+\Delta t}= \\
=\left(\begin{array}{c}
F_{0} \\
E
\end{array}\right)+\left(\begin{array}{cc}
\frac{\mathbf{T}}{\Delta t} & 0 \\
\frac{\mathbf{G}}{\Delta t} & \frac{\mathbf{L}}{\Delta t}
\end{array}\right)\left(\begin{array}{c}
A \\
\mathfrak{J}_{(\mathrm{m})}
\end{array}\right)_{t}
\end{aligned}
$$

que l'on peut facilement rendre symétrique en faisant par exemple le changement de variable

$$
\mathfrak{J}_{(\mathrm{m})}^{*}=-\frac{\Delta t}{h} \mathfrak{J}_{(\mathrm{m})},
$$

(12) s'écrivant alors sous la forme :

$$
\begin{aligned}
& \left(\begin{array}{cc}
\mathbf{S}+\frac{\mathbf{T}}{\Delta t} & \frac{h}{\Delta t} \mathbf{D} \\
\frac{h}{\Delta t} \mathbf{D}^{\mathrm{T}} & -\frac{h}{\Delta t}\left(\mathbf{R}+\frac{\mathbf{L}}{\Delta t}\right)
\end{array}\right) \times \\
& \times\left(\begin{array}{c}
A \\
\mathfrak{J}_{(\mathrm{m})}^{*}
\end{array}\right)_{t+\Delta t}=\left(\begin{array}{c}
\cdots \\
\ldots
\end{array}\right) \text {. }
\end{aligned}
$$

Le système (14) (ou celui que l'on obtient par une technique de linéarisation - Newton par exemple pour le traitement de la non-linéarité) est symétrique mais n'est pas défini positif signe - devant le bloc $\left(\mathbf{R}+\frac{\mathbf{L}}{\Delta t}\right)$. En fait les 2 équations du système (14) apparaissent comme les conditions caractérisant les points-selles d'un lagrangien. On pourrait utiliser pour résoudre ce problème, les techniques mentionnées dans $[16,17]$ mais on présente ici une adaptation de la méthode de Cholesky [18] pour la résolution de (14). Multiplions par - 1 la deuxième ligne de (14), on obtient un système anti-symétrique par blocs et dont les blocs diagonaux sont symétriques et définis positifs du type :

$$
\left(\begin{array}{lr}
\mathbf{X} & -\mathbf{B} \\
\mathbf{B}^{\mathrm{T}} & \mathbf{Z}
\end{array}\right)\left(\begin{array}{c}
A \\
\mathfrak{J}
\end{array}\right)=\left(\begin{array}{l}
\alpha \\
\beta
\end{array}\right) .
$$

On peut écrire :

$$
\left(\begin{array}{cr}
\mathbf{X} & -\mathbf{B} \\
\mathbf{B}^{\mathrm{T}} & \mathbf{Z}
\end{array}\right)=\left(\begin{array}{cc}
\mathbf{M}_{1} & O \\
\mathbf{M}_{2} & \mathbf{M}_{3}
\end{array}\right)\left(\begin{array}{cr}
\mathbf{M}_{1}^{\mathrm{T}} & -\mathbf{M}_{2}^{\mathrm{T}} \\
O & \mathbf{M}_{3}^{\mathrm{T}}
\end{array}\right) .
$$

$\mathbf{M}_{1}$ et $\mathbf{M}_{3}$ étant les matrices triangulaires inférieures correspondant à la factorisation de Cholesky de $\mathbf{X}$ et $\mathbf{Z}$ respectivement, c'est-à-dire

$$
\begin{aligned}
& \mathbf{X}=\mathbf{M}_{1} \mathbf{M}_{1}^{\mathrm{T}} \\
& \mathbf{Z}=\mathbf{M}_{3} \mathbf{M}_{3}^{\mathrm{T}} .
\end{aligned}
$$

$\mathbf{M}_{2}$ est donné par la relation

$$
\mathbf{B}=\mathbf{M}_{1} \mathbf{M}_{2}^{\mathrm{T}} \text {. }
$$

En écrivant (16) sous forme condensée on obtient donc une factorisation du type

$$
\mathbf{P}=M \tilde{M}^{\mathrm{T}}
$$

avec $\mathbf{M}$ matrice triangulaire inférieure et $\tilde{M}^{\mathrm{T}}$ matrice triangulaire supérieure, se déduisant immédiatement de $M$ par (16). Soit $n_{1}$ l'ordre de la matrice $\mathbf{X}$ et $n_{2}$ celui de la matrice $\mathbf{P}$, on peut alors à partir de (20), calculer les coefficients de la matrice $M$.

Pour $1 \leqslant i \leqslant n_{2}$ et $1 \leqslant j \leqslant n_{1}, j<i$,

$$
\begin{aligned}
m_{i i} & =\sqrt{p_{i i}-\sum_{k=1}^{i-1} m_{i k}^{2}} \\
m_{i j} & =\frac{1}{m_{j j}}\left(p_{i j}-\sum_{k=1}^{j-1} m_{i k} m_{j k}\right)
\end{aligned}
$$

et pour $n_{1}+1 \leqslant i \leqslant n_{2}$ et $n_{1}+1 \leqslant j \leqslant n_{2}, j<i$,

$$
\begin{gathered}
m_{i i}=\sqrt{p_{i i}-\sum_{k=n_{1}+1}^{i-1} m_{i k}^{2}+\sum_{k=1}^{n_{1}} m_{i k}^{2}} \\
m_{i j}=\frac{1}{m_{j j}}\left(p_{i j}-\sum_{k=n_{1}+1}^{j-1} m_{i k} m_{j k}+\right. \\
\left.\sum_{k=1}^{n_{1}} m_{i k} m_{j k}\right) .
\end{gathered}
$$

2.3 DeUXIÈME APPROCHE. - En considérant les équations électriques (3a) et (3b) (ou bien (6a) et (6b) sous forme discrétisée), on remarque que le circuit électrique est constitué des $n_{\mathrm{p}}$ branches internes (en relation directe avec le circuit magnétique, numérotées de 1 à $n_{\mathrm{p}}$ ) auxquelles on ajoute les branches du circuit extérieur (numérotées de $\left(n_{\mathrm{p}}+1\right)$ à $\left.n_{\mathrm{b}}\right)$. Lorsque les connexions sont faites, il en résulte un certain nombre de nœuds $n_{\mathrm{c}}$. Nous prenons comme éléments inconnus dans le circuit électrique, les courants de branche $\mathfrak{J}_{\left(n_{\mathrm{b}}\right)}=\left(i_{k}\right)_{k=1, n_{\mathrm{b}}}$ et les potentiels $V_{\left(n_{\mathrm{c}}\right)}=\left(V_{\ell}\right)_{\ell=1, n_{\mathrm{c}}}$ aux nœuds. En utilisant les lois d'Ohm-Kirchoff disant en substance que la somme des différences de potentiels des branches constituant une maille (chemin fermé) est nulle ainsi que, pour chaque nœud du circuit, la somme (algébrique) des courants des branches aboutissant au (ou partant du) nœud considéré. Si l'état des diodes est donné, le circuit électrique est linéaire et des considérations générales d'algèbre linéaire nous permettent d'exprimer les courants de branches internes à la machine $\mathrm{J}_{\left(n_{\mathrm{p}}\right)}=\left(i_{k}\right)_{k=1, n_{\mathrm{p}}}$ en fonction des forces électromotrices $E_{\left(n_{\mathrm{p}}\right)}=\left(e m_{j}\right)_{j=1, n_{\mathrm{p}}}=$ $-\left(\frac{\mathrm{d} \Phi_{j}}{\mathrm{~d} t}\right)_{j=1, n_{\mathrm{p}}}$, par une relation du type

$$
\mathfrak{J}_{\left(n_{\mathrm{p}}\right)}=\mathbf{Y} E_{\left(n_{\mathrm{p}}\right)}+I_{0}
$$

$\mathbf{Y}$ est une matrice $n_{\mathrm{p}} \times n_{\mathrm{p}}$, qui dans le cas présent est 
symétrique et positive (matrice d'admittance dans la théorie des réseaux électriques). La non-linéarité du circuit est traitée à l'aide d'un schéma de type prédicteur-correcteur et suivant que l'on implicite ou non le fonctionnement (commutation) des diodes, le schéma numérique d'intégration en temps devra éventuellement prévoir un retour au pas précédent. En reportant (25) dans (2) et en utilisant (7), l'équation (4) devient

$$
A_{t+\Delta t} \in \mathcal{V}, \text { solution de }
$$

$$
\begin{aligned}
& \frac{1}{\Delta t} \int_{\Omega} \sigma A_{t+\Delta t} \cdot \omega \mathrm{d} x+\int_{\Omega} \nu\left(x,\left|\operatorname{rot} A_{t+\Delta t}\right|^{2}\right) \operatorname{rot} A_{t+\Delta t} \cdot \operatorname{rot} \omega \mathrm{d} x \\
& \quad+\frac{h}{\Delta t} \sum_{i, j=1}^{n_{\mathrm{p}}} y_{i j}\left(\int_{\Omega} \psi_{i} \cdot \omega \mathrm{d} x\right)\left(\int_{\Omega} \psi_{j} \cdot A_{t+\Delta t} \mathrm{~d} x\right)=\frac{h}{\Delta t} \sum_{i, j=1}^{n_{\mathrm{p}}} y_{i j}\left(\int_{\Omega} \psi_{i} \cdot \omega \mathrm{d} x\right)\left(\int_{\Omega} \psi_{j} \cdot A_{t} \mathrm{~d} x\right) \\
& \quad+\int_{\Omega}\left(J_{0}+\sum_{i=1}^{n_{\mathrm{p}}} I_{0, i} \psi_{i}\right) \cdot \omega \mathrm{d} x+\frac{1}{\Delta t} \int_{\Omega} \sigma A_{t} \cdot \omega \mathrm{d} x \quad \forall \omega \in \mathcal{V}
\end{aligned}
$$

la matrice d'admittance $\mathbf{Y}=\left(y_{i j}\right)$ étant évaluée en $t+\Delta t$ ou $t$ selon la stratégie retenue. Cette formulation peut être vue comme une généralisation de la technique utilisée en [14]. C'est une équation intégro-différentielle en $A$, à caractère symétrique défini positif, dont une écriture matricielle pourrait être :

$$
\begin{aligned}
\left(\mathbf{S}+\frac{\mathbf{T}}{\Delta t}+\frac{h}{\Delta t} \mathbf{F Y F}^{\mathrm{T}}\right) A_{t+\Delta t} & = \\
& =F_{0}^{*}+\left(\frac{\mathbf{T}}{\Delta t}+\frac{h}{\Delta t} \mathbf{F Y F}^{\mathrm{T}}\right) A_{t} .
\end{aligned}
$$

Dans le contexte et notations de la première approche, on peut voir que la matrice d'admittance $\mathbf{Y}$ est donnée par:

$$
\mathbf{Y}=\overline{\mathbf{C N T}}\left(\mathbf{R}+\frac{\mathbf{L}}{\Delta t}\right)^{-1} \overline{\mathbf{C N T}}^{\mathrm{T}}
$$

La formulation (26) revient en fait à résoudre la deuxième équation de (12) ou (14) en fonction de $A$ et à reporter le résultat dans la première. Le système non linéaire (26) (à considérer à chaque pas de temps) peut être résolu par des méthodes de relaxation non linéaire par blocs $[14,15,19]$, combinant méthodes itératives ponctuelles et méthodes de gradient conjugué préconditionné. On peut également utiliser Newton $+\mathrm{C}$ o es y par exemp e pour résoudre (26).

\section{Applications et résultats numériques.}

3.1 DisPositif ÉleCtromagnétiQUe FIXE. - On considère dans cet exemple un système électromagnétique simple (voir [14]) dans lequel une partie massive est conductrice et donc soumise à des courants de Foucault (partie F, Fig. 1) ; ce système est alimenté par une tension de forme sinusoïdale. La géométrie et les caractéristiques magnétiques du

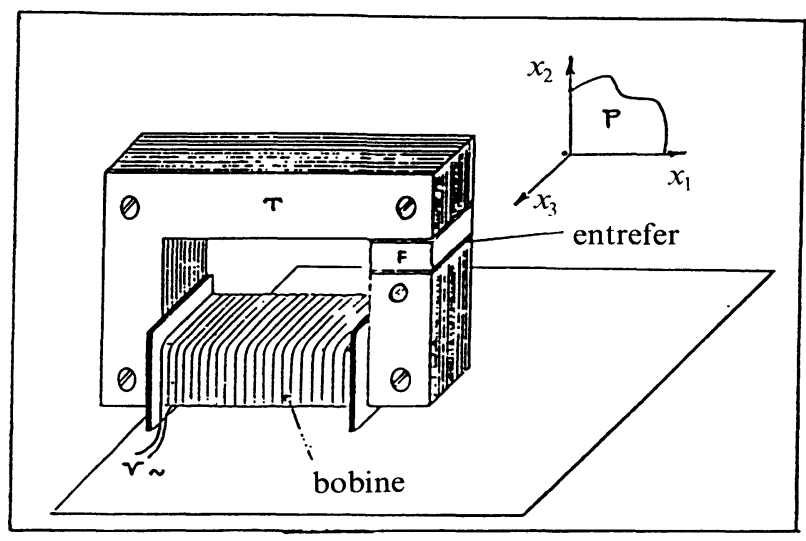

Fig. 1. - Système physique étudié.

[Studied physical system.]

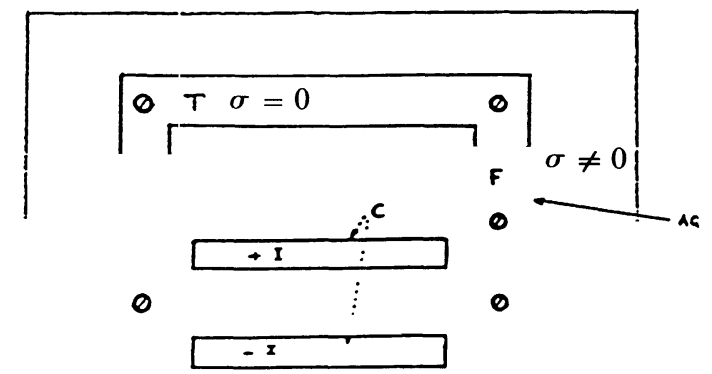

Fig. 2. - Domaine de calcul bidimensionnel.

[Bidimensional calculation domain.] 
dispositif figure 1 permettent une modélisation bidimensionnelle (contenue dans le plan $\mathbf{P}$ et représenté sur la Fig. 2). Les dimensions externes de l'armature sont $195 \mathrm{~mm} \times 120 \mathrm{~mm} \times 45 \mathrm{~mm}$. Dans le cas présent les courants de Foucault (densités) se développant dans la partie conductrice (F, fer doux) sont modélisés par (voir [14, 3])

$$
i_{\mathrm{F}}=-\sigma \frac{\partial A}{\partial t}+\frac{1}{\operatorname{aire}(F)} \int_{\mathrm{F}} \sigma \frac{\partial A}{\partial t} \mathrm{~d} x
$$

de façon à assurer la conservation du courant dans la partie $\mathbf{F}\left(\int_{\mathrm{F}} i_{\mathrm{F}} \mathrm{d} x=0\right)$.

Pour l'approximation de la caractéristique magnétique (réluctivité relative) on utilise généralement la famille de fonction $\nu_{\varepsilon, C, T, \alpha}[12]$

$$
\nu_{\varepsilon, C, T, \alpha}\left(|B|^{2}\right)=\varepsilon+(C-\varepsilon) \frac{\left(|B|^{2}\right)^{\alpha}}{\left(|B|^{2}\right)^{\alpha}+T} .
$$

Ce type de fonction a été utilisé pour la partie F. En ce qui concerne les tôles constituant l'armature $\mathbf{T}$ (ANHYSTER DS), cette famille de fonctions ne permettant pas d'avoir une variation suffisamment rapide de la réluctivité par rapport à $|B|^{2}$, nous avons utilisé la famille suivante :

$$
\nu_{c, \gamma, \alpha}\left(|B|^{2}\right)=\mathrm{e}^{-c \mathrm{e}^{-\gamma\left(\mathrm{e}^{|B|^{2}}-1\right)^{\alpha}}} .
$$

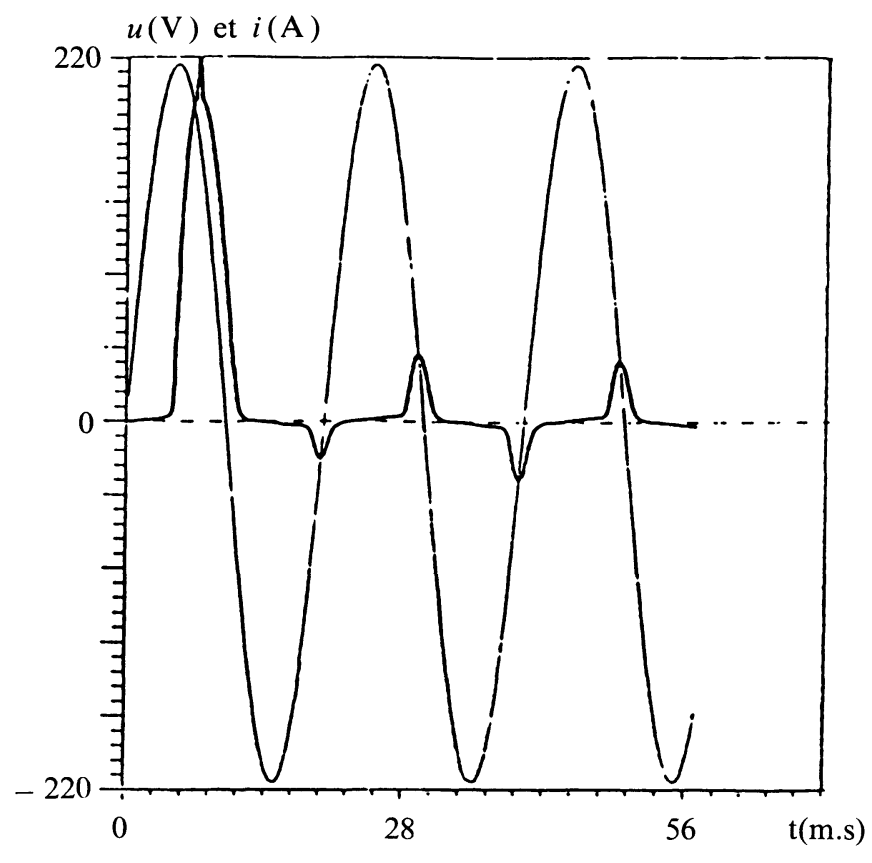

Fig. 3. - Forme d'onde de la tension d'alimentation (courbe 1) et du courant calculé dans la bobine (courbe 2).

[Fed voltage wave form (C1) and calculated current in the winding $(\mathrm{C} 2)$.]

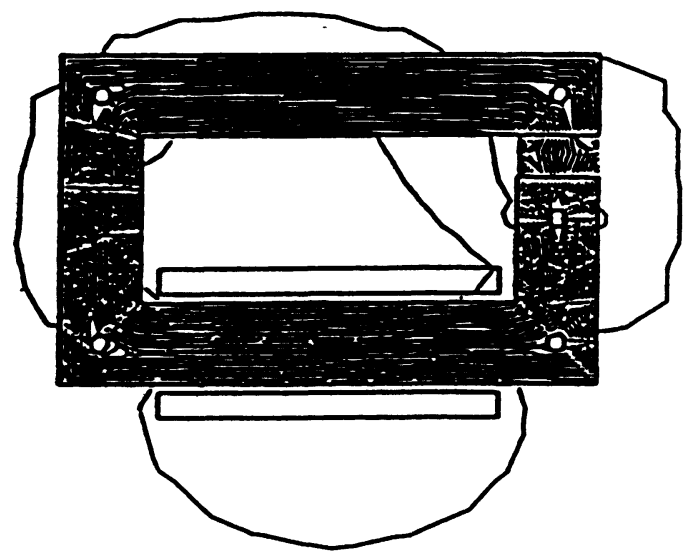

Fig. 4. - Tracé des lignes de champ dans la bobine pour un point de fonctionnement où le courant est maximum.

[Flux distribution in the winding for an operating point corresponding to the maximum current.]

Un lissage par moindres carrés permet de trouver des valeurs pour les paramètres; à titre d'exemple pour (29b) on a obtenu $c=10,6222, \alpha=4,4551$, $\gamma=0,09979$. Dans cette application le circuit électrique se réduit à une maille. Si $\mathbf{R}$ représente la résistance totale du circuit, la matrice d'admittance définie en (25) ou (27), dans le cas où l'on ne tient pas compte des inductances de fuite, est tout simplement le scalaire $\frac{1}{\mathbf{R}}$.

On peut voir sur la figure 3 la tension d'alimentation et la forme d'onde calculée du courant dans la bobine. Les calculs représentés sur cette figure ont été faits avec une discrétisation temporelle $T / \Delta t=$ 100. Sur la figure 4 on peut voir le tracé des lignes de champ et l'influence des courants de Foucault dans la partie conductrice. Ce tracé correspond à un point de fonctionnement où le courant est maximum. Les résultats numériques présentés ici, obtenus via la deuxième approche (mais on aurait pu évidemment utiliser la première), ont montré une bonne concordance avec les mesures expérimentales.

3.2 MACHINE SYNCHRONE À AIMANTS PERMANENTS. - Pour cette machine modélisée avec la première approche, nous avons simulé son fonctionnement en générateur associé à un redresseur. Sa puissance apparente est de $6,2 \mathrm{kVA}$ à la vitesse de $30000 \mathrm{tr} / \mathrm{min}$ pour une tension de $500 \mathrm{~V}$. Cette machine possède quatre pôles et est équipée d'aimants permanents $\left(B_{\mathrm{r}}=0,82 \mathrm{~T}\right)$; le rayon moyen de l'entrefer est de $39,5 \mathrm{~mm}$ et sa longueur active de $50 \mathrm{~mm}$. Pour la modélisation, les caractéristiques des matériaux ferromagnétiques sont représentées à l'aide de la relation (29a). La figure 5 montre le tracé des lignes de champ pour un point de fonctionnement à vide et une position relative du rotor par rapport au stator de $\pi / 4$. On trouvera sur 
la figure 6, le schéma du montage étudié. A titre d'exemple nous avons simulé l'établissement du courant dans la charge pour un point de fonctionne-

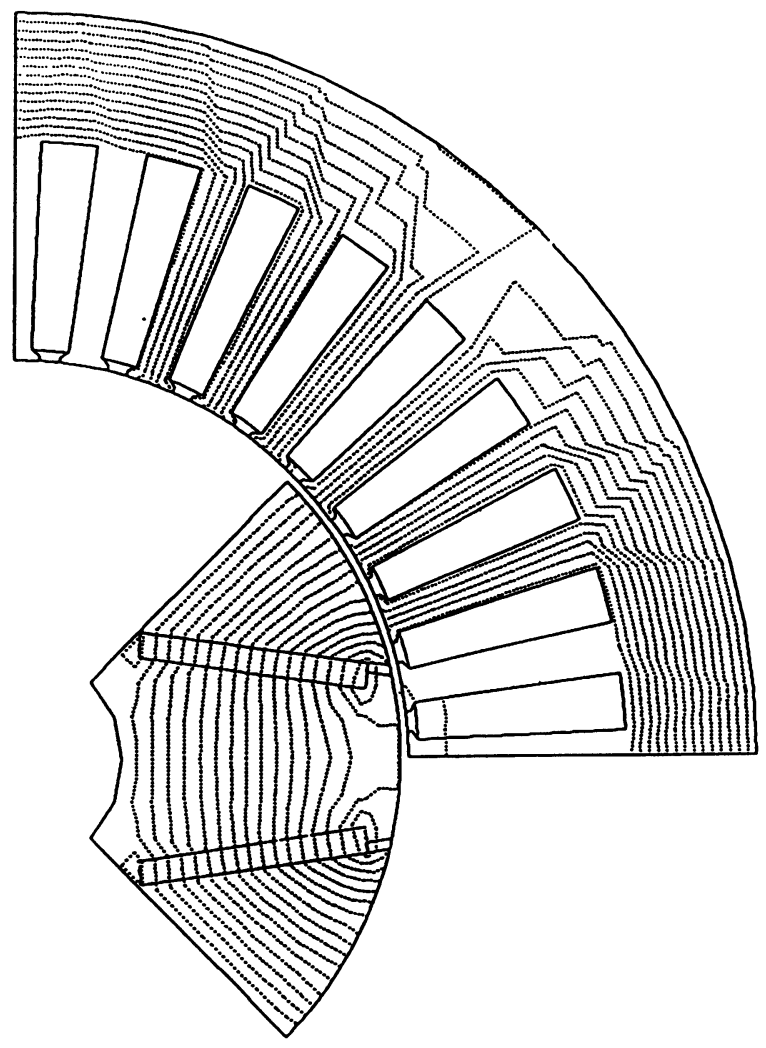

Fig. 5. - Tracé des lignes de champ dans la section modélisée pour un point de fonctionnement à vide et une position relative du rotor par rapport au stator de $\pi / 4$.

[Flux distribution in the studied domain at no-load for a relative position of the rotor and the stator of $\pi / 4$.]

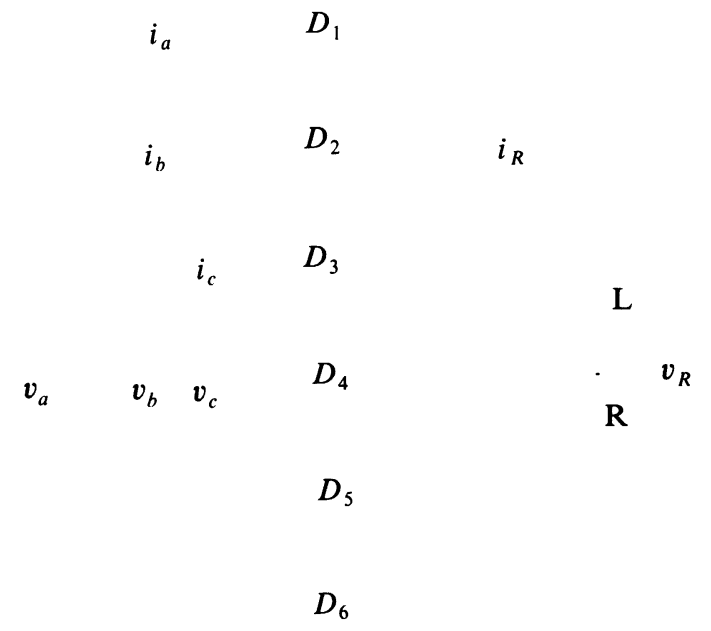

Fig. 6. - Montage étudié (redressement double alternance).

[Studied system (bridge rectifier).] ment correspondant à $R=15 \Omega, L=0,13 \mathrm{~h}$ et $N=3000 \mathrm{tr} / \mathrm{min}$. La figure 7 montre l'évolution du courant en fonction du temps, dans la charge (courbe 1) et dans une phase de la machine (courbe 2). Pour les mêmes conditions de fonction-

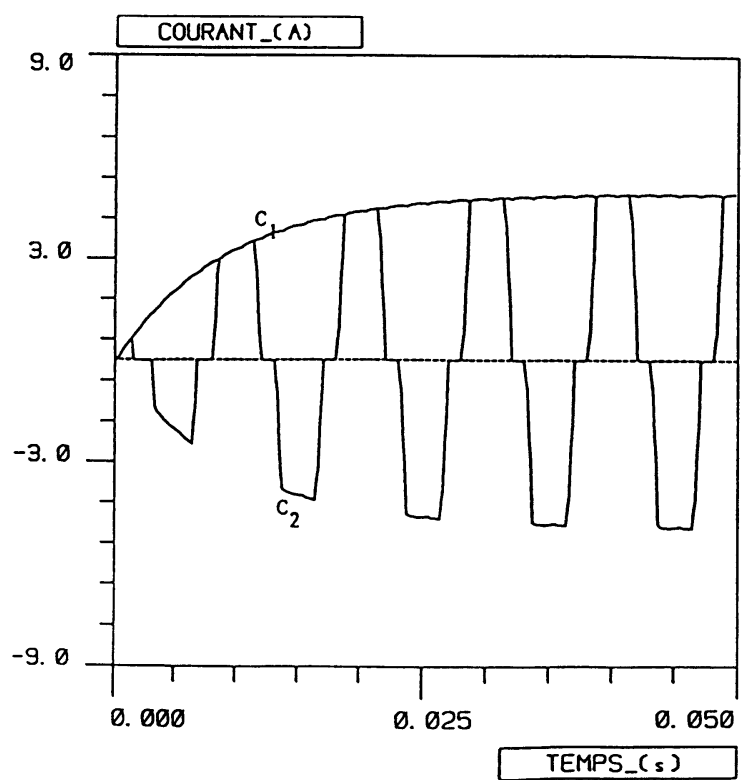

Fig. 7. - Redressement double alleinunnce : évolution du courant calculé dans la charge et une phase de la machine.

[Double rectifier : evolution of the calculated load current and the armature current.]

9.0

\section{. COURANT_(A)}

3. 0

$-3.0$

$-9.0$

0.000

0.085

0.010

TEMPS_(s)

Fig. 8. - Redressement double alternance : forme d'onde calculée du courant dans une phase en régime permanent.

[Double rectifier: calculated current wave form in the armature at steady state.] 


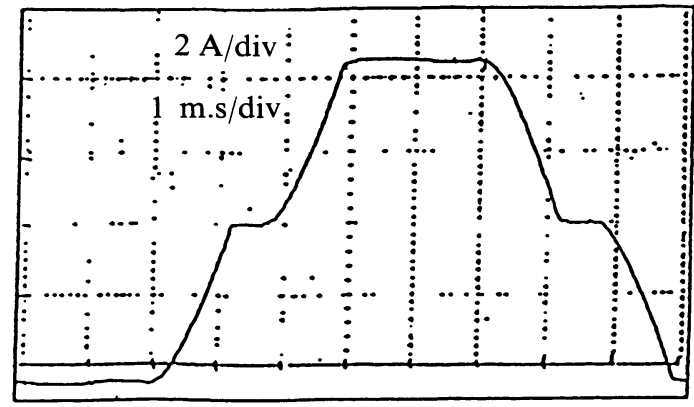

Fig. 9. - Redressement double alternance : forme d'onde obtenue expérimentalement du courant dans une phase en régime permanent.

[Double rectifier : experimental current wave form in the armature at steady state.]

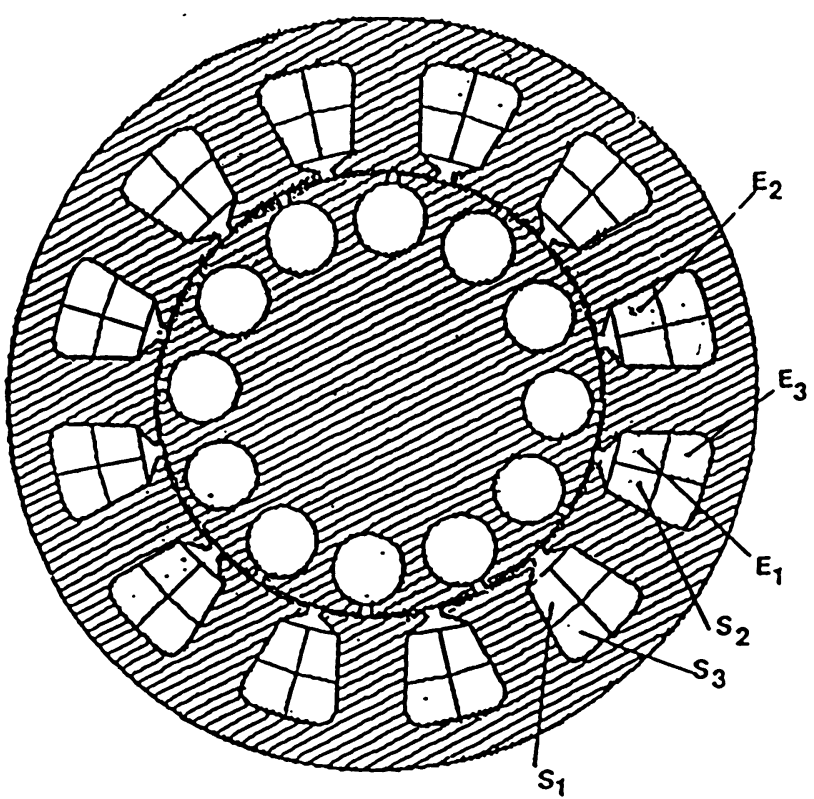

nement, la figure 8 montre la forme d'onde calculée du courant dans une phase de la machine en régime permanent et la figure 9 celle obtenue expérimentalement.

3.3 Alternateur À CAGE. - Cet alternateur dont le fonctionnement n'est pas classique a été spécialement conçu par la société VALEO et les deux approches proposées ont été testées simultanément. Cette machine dont la section transversale $\left(\phi_{\text {ext }}=\right.$ $80 \mathrm{~mm}$ ) et le schéma de bobinage sont montrés figure 10, comprend au stator un enroulement d'excitation et 2 enroulements d'induits répartis dans 12 encoches. Quant au rotor, il est constitué de 12 barres en court-circuit. Etant donné les répétitivités géométriques et électriques, le domaine de calcul peut se réduire à $1 / 12^{\mathrm{e}}$ de la machine. Les caractéris-
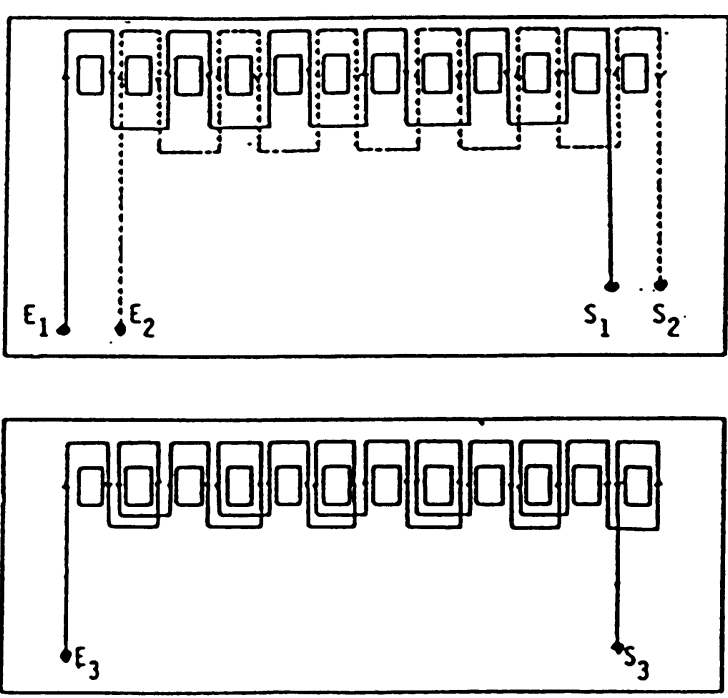

Fig. 10. - Coupe transversale de l'alternateur à cage $\left(\phi_{\text {ext }}=80 \mathrm{~mm}\right)$ et schéma de bobinage.

[Cross section of the studied alternator and circuit diagrams of the different windings.]

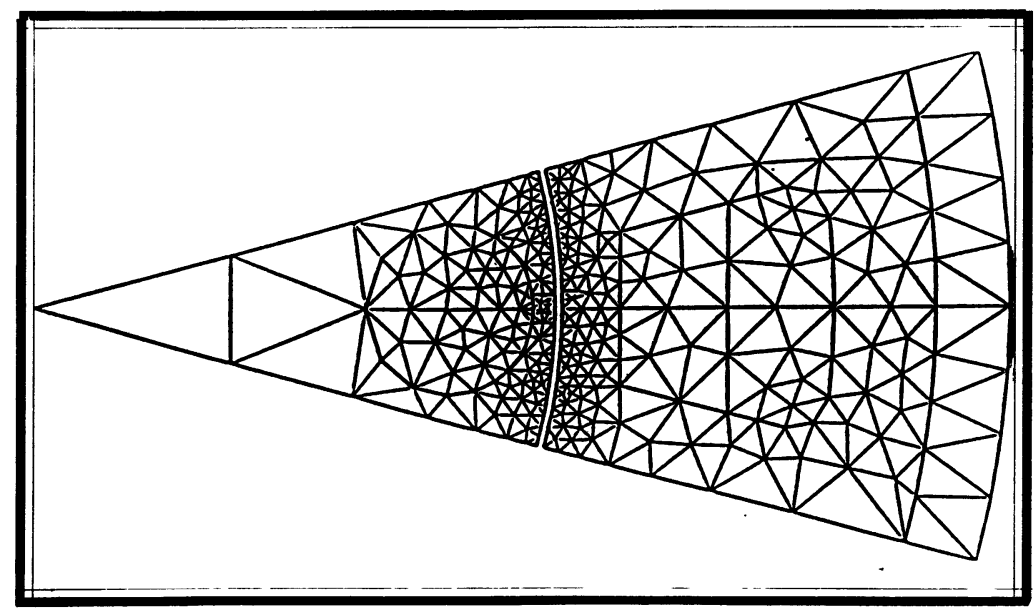

Fig. 11. - Maillage du domaine étudié (cas du macroélément).

[Studied domain mesh (in the case of a macro-element).] 
tiques des matériaux magnétiques sont approchées en utilisant la relation (29a). Le maillage utilisé pour la première approche est représenté sur la figure 11 (la prise en compte du mouvement est faite en utilisant un macro-élément $[7,10])$. La figure 12 représente un «zoom » de la région entrefer utilisée dans la deuxième approche (la prise en compte du mouvement est faite en utilisant la ligne de glissement $[6,12])$. Différents calculs ont été effectués sur cette machine en utilisant les deux approches proposées (celles-ci ont donné des résultats identiques). A

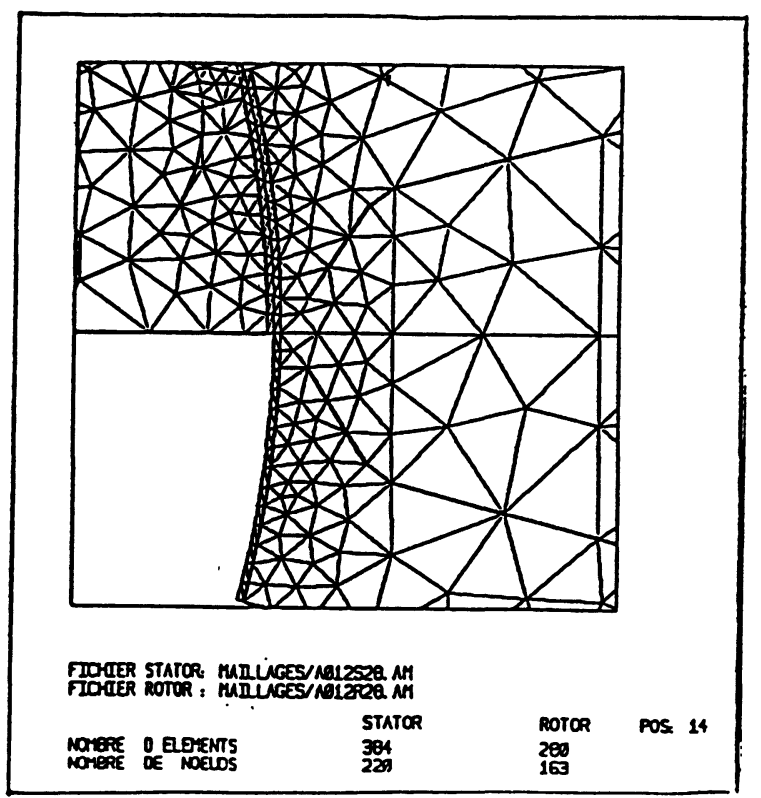

Fig. 12. - Zoom autour de l'entrefer du maillage (cas de la ligne de glissement).

[Mesh zoom near the air gap (in the case of a displacement line).]

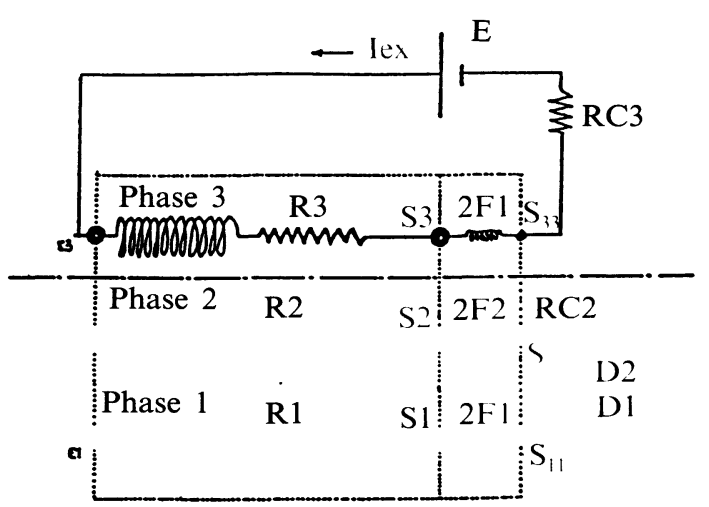

$\mathbf{R}$

Fig. 13. - Schéma du circuit électrique.

[Electric circuit scheme.]

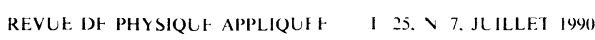

titre d'exemple nous allons présenter les résultats obtenus lorsque l'alternateur est associé à un redresseur (voir schéma électrique Fig. 13). Pour un point de fonctionnement correspondant à une vitesse de rotation de $3000 \mathrm{tr} / \mathrm{min}$ et une tension $E=15 \mathrm{~V}$, les figures 14,15 et 16 montrent les résultats obtenus à partir de la simulation (traits pointillés) et de l'expérience (traits pleins), pour respectivement le courant dans le circuit d'excitation, la tension aux bornes des phases et le courant dans la résistance $R$.

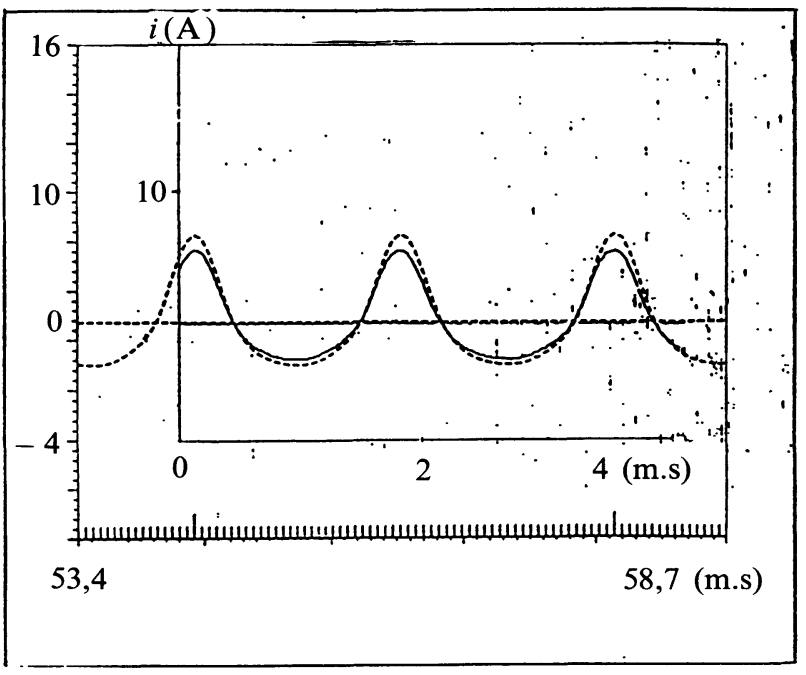

Fig. 14. - Forme d'onde du courant calculée (trait pointillé) et relevée expérimentalement (trait plein) dans l'enroulement du circuit d'excitation.

[Calculated current wave form (broken line) and measurement one (black line) in the excitation circuit.]

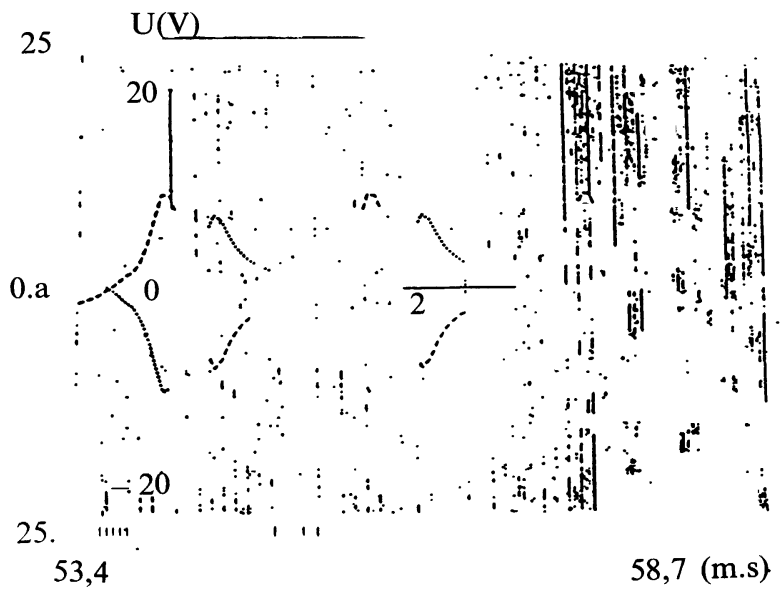

Fig. 15. - Forme d'onde de la tension calculée (trait pointillé) et relevée expérimentalement (trait plein) aux bornes des phases de l'induit.

[Calculated phase voltage wave form (broken line) and measurement on (black line). 


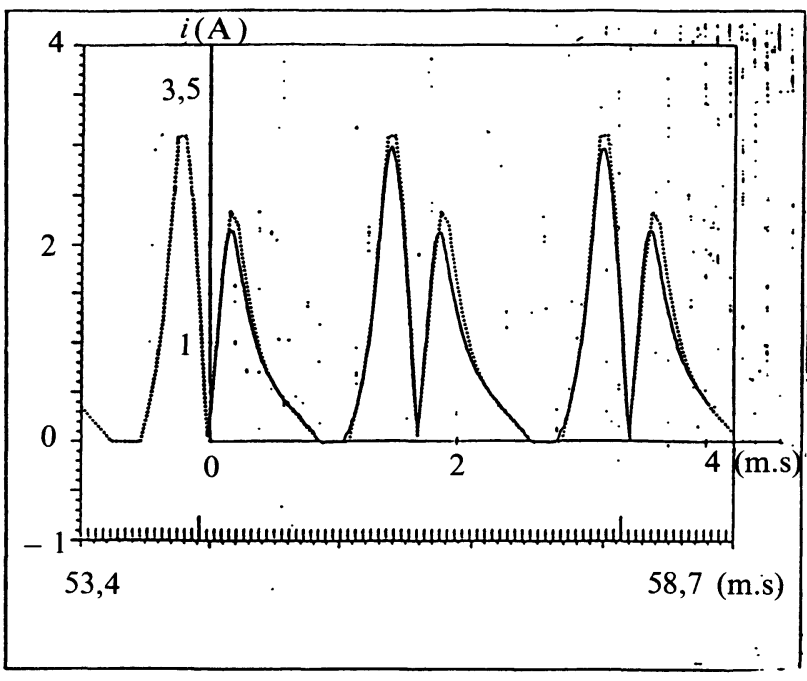

Fig. 16. - Forme d'onde du courant calculée (trait pointillé) et relevée expérimentalement (trait plein) dans la charge.

[Calculated load current wave form (broken line) and measurement one (black line).]

Les calculs ont été effectués en prenant une tension de seuil pour les diodes : $E_{\mathrm{s}}=0,8 \mathrm{~V}$; on a pris comme longueur active des conducteurs lors du calcul des flux, l'épaisseur du stator $(50 \mathrm{~mm})$. Pour le même point de fonctionnement on peut voir sur la figure 17 l'établissement du courant d'alimentation ainsi que l'évolution de la DDP aux bornes $\left(V_{\mathrm{S}_{3}}-V_{\mathrm{E}_{3}}\right)$.

\section{Conclusion.}

Dans cette communication, nous avons développé deux approches qui permettent de modéliser des systèmes électromagnétiques en couplant les équations magnétiques et électriques. Dans la première approche, les inconnues sont les valeurs nodales du potentiel vecteur et les courants de mailles. Dans la seconde, on conserve uniquement le potentiel vecteur comme inconnue en exprimant les grandeurs

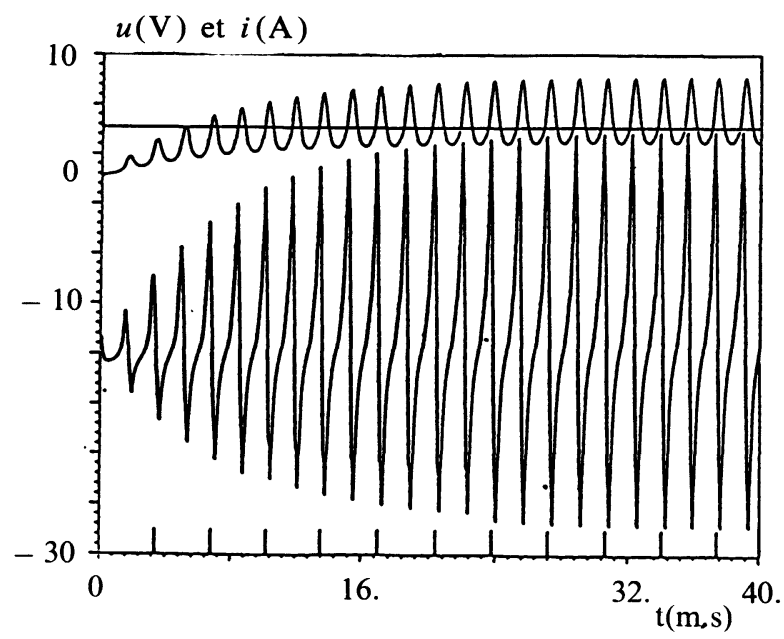

Fig. 17. - Evolution du courant d'excitation et de la tension aux bornes du circuit (simulation numérique).

[Calculated transient voltage and current in the excitation circuit.]

électriques en fonction de celui-ci et en reportant le résultat dans l'équation magnétique. Les différences entre ces deux approches ont été étudiées ainsi que l'influence sur le système final à résoudre. Ces deux techniques utilisées pour modéliser un même problème ont donné des résultats identiques et une bonne concordance avec les résultats expérimentaux.

Comme exemples d'applications, nous avons présenté les résultats obtenus pour une bobine à noyau ferromagnétique, une machine synchrone à aimants permanents et un alternateur à cage. L'ensemble de ces résultats montre les possibilités et la validité des deux modèles proposés.

\section{Remerciements.}

Les auteurs remercient la Société VALEO pour le support financier, les études expérimentales et la collaboration technique relatives à l'étude de l'alternateur à cage.

\section{Bibliographie}

[1] Piriou F., RAzek A., Calculation of saturated inductances for numerical simulation of synchronous machines, IEEE Mag. 19 (1983) 2628-2631.

[2] Sabonnadière J. C., Méthodes de calcul numérique en électrotechnique : application aux machines électriques, RGE (1982) 645-657.

[3] Biedinger J. M., Contribution à la modélisation numérique des machines électriques mobiles, Thèse $3^{\mathrm{e}}$ cycle, Université de Compiègne (1981).

[4] Shen D., Meunier G., Coulomb J. L., SabonnaDIÈRE J. C., Solution of magnetic field and electrical circuit combined problem, IEEE Mag. 21 (1985) 2288-2291.

[5] Strangas E. G., Coupling the circuit equations to the non-linear time dependent field solution in inverter driven induction motors, IEEE Mag. 21 (1985) 2408-2411.

[6] Preston T. W., Reece A. B. J., SAngha P. S., Induction motor analysis by time-stepping techniques, IEEE Mag. 24 (1988) 471-474.

[7] Bouillault F., Razek A., Prise en compte du mouvement dans la détermination numérique des courants de Foucault dans une structure 
électromagnétique, Revue Phys. Appl. 18 (1983) 103-106.

[8] Bouillault F., Contribution à la modélisation de systèmes électromagnétiques en mouvement comportant des parties ferromagnétiques massives. Thèse $3^{\mathrm{e}}$ cycle, Université Paris 6 (1984).

[9] Davat B., Ren Z., Lajoie-Mazenc M., The movement in field modeling, IEEE Mag. 21 (1985) 2296-2298.

[10] Feliachi M., Contribution au calcul du champ électromagnétique par la méthode des éléments finis en vue d'une modélisation numérique des machines électriques, Thèse de Docteur-Ingénieur, Paris (1981).

[11] RazeK A., Coulomb J. L., Feliachi M., SabonnaDIÈRE J. C., Conception of an air gap element for dynamic analysis of the electromagnetic field in electric machines, IEEE Mag. 18 (1982) 655659.

[12] Marrocco A., Analyse numérique de problèmes d'électrotechnique, Ann. Sc. Math. Québec 1 (1977) 271-296.

[13] Piriou F., RAzeK A., Coupling of saturated electromagnetic systems to non-linear power electronic devices, IEEE Mag. 24 (1988) 274-277.
[14] Marrocco A., Computation of the coil and eddy currents in a voltage supplied system, Conférence Franco-Soviétique, Novosibirsk (juin 1981).

[15] Marrocco A., Simulations numériques dans la fabrication des circuits à semiconducteurs, Rapport de recherche INRIA, $n^{\circ} 305$ (mai 1984).

[16] ForTin M., GLOwINSKI R., Méthodes de lagrangien augmenté. Applications à la résolution numérique de problèmes aux limites (Dunod). Collection Méthodes Mathématiques de l'Informatique 9 (1982).

[17] Glowinski R., Lions J. L., Trémolières R., Numerical analysis of variational inequalities (North-Holland). Studies in Mathematics and its Applications 8 (1981).

[18] Long W., Piriou F., Razek A., An adapted Cholesky decomposition method, to appear in Compel.

[19] Hecht F., Marrocco A., Piriou F., Razek A., Modélisation électromagnétique d'un alternateur couplé à un circuit extérieur, Proceedings of Team Workshop and Meeting on the Applications of Eddy-Currents Computations, Bièvres (March 1989) 265-278. 